\title{
Article \\ Characterization of Ex Vivo Nonmelanoma Skin Tissue Using Raman Spectroscopy
}

\author{
Hieu T. M. Nguyen ${ }^{1}{ }^{\oplus}$, Yao Zhang ${ }^{1}$, Austin J. Moy ${ }^{1}$, Xu Feng ${ }^{1}{ }^{\circledR}$, Katherine R. Sebastian ${ }^{2}$, Jason S. Reichenberg ${ }^{2}$, \\ Matthew C. Fox ${ }^{2}$, Mia K. Markey ${ }^{1,3}$ and James W. Tunnell ${ }^{1, *(\mathbb{D})}$ \\ 1 Department of Biomedical Engineering, The University of Texas at Austin, 107 W. Dean Keeton Street C0800, \\ Austin, TX 78712, USA; hieung@utexas.edu (H.T.M.N.); zhangyao1994@utexas.edu (Y.Z.); \\ austin.moy@gmail.com (A.J.M.); xu.feng@utexas.edu (X.F.); mia.markey@utexas.edu (M.K.M.) \\ 2 Department of Internal Medicine, Dell Medical School, The University of Texas at Austin, 1701 Trinity Street \\ Z0900, Austin, TX 78712, USA; KrSebastian@seton.org (K.R.S.); jreichenberg@seton.org (J.S.R.); \\ MCFox@seton.org (M.C.F.) \\ 3 Department of Imaging Physics, The University of Texas MD Anderson Cancer Center, 1515 Holcombe Blvd, \\ Houston, TX 77030, USA \\ * Correspondence: jtunnell@mail.utexas.edu
}

check for updates

Citation: Nguyen, H.T.M.; Zhang, Y.; Moy, A.J.; Feng, X.; Sebastian, K.R.; Reichenberg, J.S.; Fox, M.C.; Markey, M.K.; Tunnell, J.W. Characterization of Ex Vivo Nonmelanoma Skin Tissue Using Raman Spectroscopy. Photonics 2021, 8, 282. https://doi.org/ $10.3390 /$ photonics 8070282

Received: 14 June 2021

Accepted: 14 July 2021

Published: 16 July 2021

Publisher's Note: MDPI stays neutral with regard to jurisdictional claims in published maps and institutional affiliations.

Copyright: (C) 2021 by the authors Licensee MDPI, Basel, Switzerland. This article is an open access article distributed under the terms and conditions of the Creative Commons Attribution (CC BY) license (https:// creativecommons.org/licenses/by/ $4.0 /)$.

\begin{abstract}
Raman spectroscopy has shown great potential in detecting nonmelanoma skin cancer accurately and quickly; however, little direct evidence exists on the sensitivity of measurements to the underlying anatomy. Here, we aimed to correlate Raman measurements directly to the underlying tissue anatomy. We acquired Raman spectra of ex vivo skin tissue from 25 patients undergoing Mohs surgery with a fiber probe. We utilized a previously developed biophysical model to extract key biomarkers in the skin from the Raman spectra. We then examined the correlations between the biomarkers and the major skin structures (including the dermis, sebaceous glands, hair follicles, fat, and two types of nonmelanoma skin cancer-basal cell carcinoma (BCC) and squamous cell carcinoma (SCC)). SCC had a significantly different concentration of keratin, collagen, and nucleic acid than normal structures, while ceramide differentiated BCC from normal structures. Our findings identified the key proteins, lipids, and nucleic acids that discriminate nonmelanoma tumors and healthy skin using Raman spectroscopy. These markers may be promising surgical guidance tools for detecting tumors in resection margins.
\end{abstract}

Keywords: Raman spectroscopy; nonmelanoma skin cancer; biophysical model

\section{Introduction}

Raman spectroscopy offers a non-destructive, fast, and label-free optical approach to assess tumor margins. Several studies have reported the potential of tumor margin assessment using Raman spectroscopy in numerous types of cancer, including skin [1-5], oral cavity [6,7], breast [8,9], brain [10,11], lung [12,13], and stomach [14,15]. These studies can be categorized into two main instrument approaches: handheld fiber probe for singlepoint sampling and scanning microscopy for optical imaging. While Raman microscopy offers high resolution and good diagnostic accuracy, current systems' acquisition times are still too long for clinical use. The fiber probe approach, on the other hand, can offer a quick diagnostic method and provide timely assistance in evaluating tumor margins.

Previous studies have shown encouraging results for classifying nonmelanoma skin cancer versus normal tissue with Raman fiber probes [16-21]. Several studies have reported high sensitivity (63-100\%) and specificity (83-91\%) for discriminating the major types of nonmelanoma skin cancer, basal cell carcinoma (BCC), and squamous cell carcinoma (SCC) from normal skin in vivo [16,21,22] and ex vivo [23,24]. Though high diagnostic accuracy has been demonstrated in these studies, most used statistical algorithms to transform Raman spectra to a few significant dimensions, including principal component 
analysis [16,17,20,21], linear discriminant analysis [23], and maximum representation and discrimination feature $[18,19]$. As a result, the approach did not elucidate the nature of the biochemical processes responsible for the spectral differences. Furthermore, little direct evidence exists on the sensitivity of the measurements to the underlying anatomy or the role of the healthy skin structures in tumor classification. Indeed, many of the proteins, lipids, and nucleic acids that can be sensed using Raman spectroscopy are present in both normal and tumor tissues.

Our study is the first report to correlate Raman fiber probe measurements directly with the underlying skin tissue anatomy. We characterized biomarkers in ex vivo nonmelanoma skin tissue using a biophysical human skin cancer model developed previously by our group. This model infers the skin's biochemical makeup, such as collagen, ceramide, elastin, keratin, nucleus, triolein, and water from its Raman spectrum [25]. We first correlated the biomarkers to the proportion of skin structures within the fiber probe sampling area. We observed several correlations consistent with our previous work on the biophysical basis for tumor assessment of skin based on the Raman microscopy approach [24]. The sampling area of the microscopy approach was on the order of $1 \mu \mathrm{m}^{2}$, while the probe's sampling area in this study was $1 \mathrm{~mm}^{2}$. As a result, we observed a lesser sensitivity in this study due to more heterogeneity within the sampling volume. Though Raman microscopy offered a higher sensitivity in detecting cancer, this approach can be lengthy (5-6 hours for one skin tissue sample). Here, our study focused on the fiber probe approach, which can potentially provide the Mohs physician with quick guidance to remove the cancer tissue during the surgery.

\section{Materials and Methods}

\subsection{Instrumentation}

The instrument setup has been described in detail in our previous studies $[26,27]$. Briefly, an $830 \mathrm{~nm}$ diode laser (Ondax, max power $500 \mathrm{~mW}$ ) was coupled to an optical fiber that was bundled into a custom-designed handheld probe [28]. The laser excitation power at the surface of the tissue sample was around $60 \mathrm{~mW}$. The probe was placed in contact with samples during measurement to excite and collect a Raman signal from a sampling area of $1 \mathrm{~mm}^{2}$ (a sampling depth of $1 \mathrm{~mm}$ ) directly beneath the probe. The Raman fiber probe's sampling depth was studied previously by our group [28,29]. Briefly, we acquired the spectra of a Raman reference (acetaminophen) submerged in a liquid phantom. This phantom contained a 1- $\mu \mathrm{m}$ diameter microbead solution that represents the absorption and scattering of human tissue (absorption coefficient of $1 \mathrm{~cm}^{-1}$ and reduced scattering coefficient of $10 \mathrm{~cm}^{-1}$ at $630 \mathrm{~nm}$ ). As the probe moved vertically from the phantom, we monitored select Raman peaks' spectral intensity at $1324 \mathrm{~cm}^{-1}$ and defined the sampling depth as 50\% attenuation from this sigmoid relationship between spectral intensity and depth. The Raman signal from the skin was collected from the seven fibers on the probe and delivered to a custom-configured Raman spectrometer with a spectral resolution of $18 \mathrm{~cm}^{-1}$ (full width at half maximum) for a $100 \mu \mathrm{m}$ slit (Stroker model from Wasatch Photonics). We used LabVIEW (National Instruments) to control laser exposure time and the spectrometer's integration time. The integration time was set at 5 seconds for Raman signal acquisition.

\subsection{Skin Samples}

The skin tissue samples were collected following an approved Institutional Review Board (IRB) protocol at The University of Texas at Austin and the Seton Healthcare Family (\#2013-07-0008). A total of 34 excised tissue blocks were collected from 25 patients who had undergone Mohs surgery, the current standard of care for nonmelanoma skin cancer [30], at the Austin Dermatologic Surgery Center. The Mohs surgical procedure involves excision of the debulked tumor with a narrow margin. This tissue layer is then examined using en face frozen section microscopic analysis to determine the presence of residual tumor cells. If tumor cells remain at surgical margins, the Mohs surgeon anesthetizes the patient's 
skin again, removes another layer of tissue, and again examines excised tissue margins under the microscope after histopathologic processing. This process iterates until the surgical margins are clear. The skin samples' thickness is in the range of $1-4 \mathrm{~mm}$. We acquired 82 measurements on these tissue blocks from the excised surface. Following each Raman measurement, we marked the measurement site with a green dye for correlation in histological sections. The tissue was sectioned along the light transport direction into tissue at the center of the green dye marker. The tissue was then processed for standard, formalin-fixed hematoxylin and eosin (H\&E) histology. A board-certified dermatologist and fellowship-trained Mohs micrographic surgeon (Dr. Fox) identified major tissue structures such as dermis (Der), sebaceous glands (SG), hair follicles (HF), fat, BCC tumor, and SCC tumor within the $1 \mathrm{~mm}^{2}$ sampling area (highlighted by the white dashed box) immediately below the measurement location (Figure 1). We used ImageJ (NIH) to quantify the area of these skin structures for all the measurements.
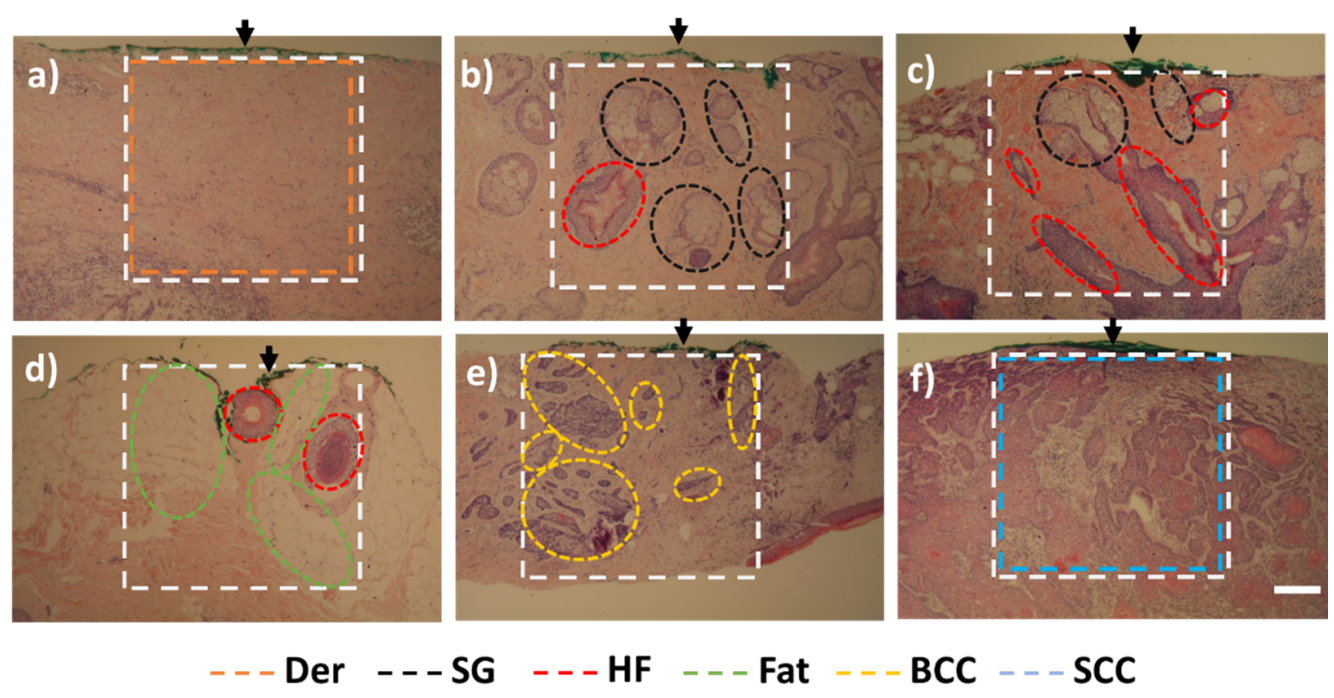

Fat
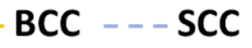

Figure 1. Examples of tissue structure within the sampling area of the fiber probe with the main area of (a) dermis (Der), (b) sebaceous glands (SG), (c) hair follicles (HF), (d) fat, (e) BCC tumor, and (f) SCC tumor. The green dye on skin tissue (marked by the black arrow) shows the location of Raman acquisition, and the white dashed box highlights the sampling area of $1 \mathrm{~mm}^{2}$. Scale bar: $200 \mu \mathrm{m}$.

Table 1 provides a summary of the pathological assessment of all the measurements. We defined a sample as cancer (BCC or SCC) when the dermatologist identified tumors within the sampling area. Sampling areas containing healthy skin structures such as hair follicles, sebaceous glands, fat, and dermis were categorized as normal skin. Among the 82 measurements, 18 were BCC, 12 were SCC, and 52 were normal. Among the 52 normal skin measurements, all samples had dermis, 27 samples with fat, 16 samples with sebaceous glands, and 14 samples with hair follicles. We reported normal skin patients only when all the measurement sites were confirmed as normal skin by histopathology. If one of the measurements was confirmed as a tumor, the patient was counted as with a tumor.

Table 1. Summary of samples' pathological assessment.

\begin{tabular}{cccc}
\hline & Normal & BCC & SCC \\
\hline Number of measurements & 52 & 18 & 12 \\
Number of patients & 11 & 8 & 6 \\
\hline
\end{tabular}

\subsection{Raman Signal Processing and Extraction of Biomarkers}

The processing procedure for raw Raman spectra included wavenumber calibration, dark noise background removal, cosmic ray removal, smoothing, and a fifth-order poly- 
nomial fitting to remove tissue fluorescence background, as described in our previous studies [17]. The spectral intensity response was calibrated using an LS-1-CAL calibrated tungsten halogen lamp (Ocean Optics, Dunedin, FL, USA).

We applied a previously developed biophysical model to the data to extract the biomarkers in skin samples [25]. Our model was built based on elemental Raman spectra of key components in the skin and used linear fitting to extract these components' contribution. For the skin samples collected in this study, we assumed seven key Raman active components for model fitting, including collagen, elastin, triolein, keratin, nucleus, ceramide, and water. Melanin was minimal within the dermis of the skin in these samples; hence it was not included in the model. All the model components were extracted from human skin and contained rich biochemical and structural information. For instance, collagen and elastin were the major constituents in the dermal extracellular matrix. The nucleus represented the nuclear material in the cell. Ceramide was an essential constituent in sebaceous lipid, while triolein existed in large amounts in subcutaneous fat.

\subsection{Statistical Analysis}

To compare the difference between groups of samples, we used R (RStudio 2018) to perform an analysis variance (ANOVA) combined with Tukey's test as a post hoc analysis.

\section{Results and Discussion}

The skin biophysical contribution within the sampling area was first extracted using our previously developed Raman biophysical skin model [25]. Figure 2 shows the model fit to Raman spectra from samples with dominant skin structures of BCC tumor, dermis, fat, SCC tumor, hair follicles, and sebaceous glands. The model fits the Raman skin spectra well, with mean residuals below $5 \%$. This procedure's outputs were fit coefficients of seven biocomponents, including collagen, elastin, triolein, keratin, nucleus, ceramide, and water representing each component in the sampling area. Even though the Raman spectra of different groups of skin tissue appear to be similar, several studies have shown that Raman signals can be resolved to a multitude of contributions from various biophysical tissue constituents [24,31,32]. Feng et al. [25] used Raman imaging and multivariate curve resolution analysis to determine the Raman spectra of the individual in situ skin constituents, which were combined linearly to fit in vivo human skin spectra. LarraonaPuy et al. [31,32] used linear discriminant analysis to build a multivariate supervised classification model of normal skin and tumors. These methods could resolve the slight differences in Raman spectra to statistical differences between normal skin and tumors.
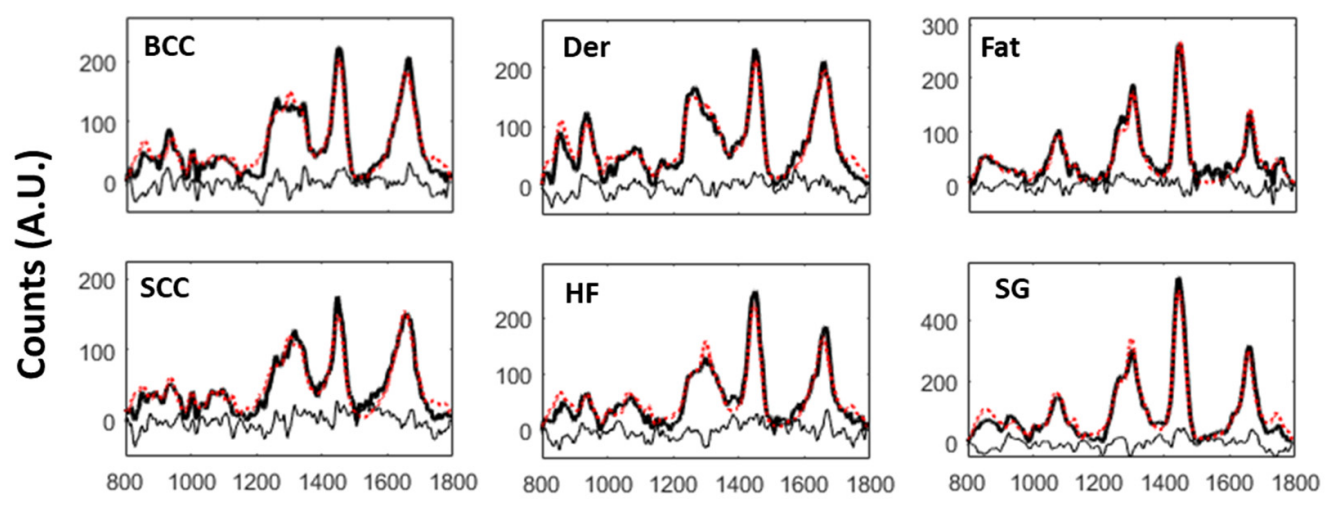

Wavenumber $\left(\mathrm{cm}^{-1}\right)$

Figure 2. Examples of fitting of Raman spectra of skin samples that are dominant with BCC tumor, dermis (Der), Fat, SCC tumor, hair follicles (HF), and sebaceous glands (SG). Black bold solid lines represent Raman signal from skin samples, red dotted lines show model fitting, and black lines at the bottom are residuals from fitting. 
In order to identify the key biomarkers associated with the various skin structures, we computed the Pearson correlation coefficients (PCC) between each biomarker contribution extracted from the Raman spectra versus the area of each skin structure from the histology slices (Table 2). We highlighted the pairs that resulted in strong correlation (PCC $>0.6$, dark gray), moderate correlation $(0.4<\mathrm{PCC}<0.6$, gray), and weak correlation $(0.2<$ $\mathrm{PCC}<0.4$, light gray). Figure 3 highlights a few key correlations. For demonstrating the correlation coefficients computed in Table 2, we divided the percentage of tissue structure equally in three sections (low, medium, and high percentage) to visualize the trends of the contribution of biomarkers across these three sections. The SCC tumor area was positively correlated with the keratin in the sampling area (Figure 3a). Keratin is a fibrous structural protein produced by keratinocytes and is abundant in the normal epidermis and dermis. The positive correlation of keratin in SCC tumors may be attributed to large keratinization areas in response to malignant cells [33]. The SCC tumor area negatively correlated with collagen in the sampling tissue (Figure $3 b$ ). Collagen is a major component in the extracellular matrix, and as SCC tumors progress, matrix metalloproteinases are involved in the fragmentation of the extracellular matrix, which eventually leads to the breakdown of collagen $[34,35]$. We observed a negative correlation between BCC and ceramide (Figure 3c). Ceramide, the main stratum corneum polar lipid, plays a crucial role in skin barrier function and epidermal differentiation. The lack of ceramide in the skin might result in the mutation of basal cells in the epidermis and initiation of BCC growth [36].

Table 2. Pearson correlation coefficients between biocomponents and tissue structures.

\begin{tabular}{ccccccc}
\hline & Fat & SG & HF & Der & BCC & SCC \\
\hline Triolein & 0.61 & -0.07 & -0.12 & -0.08 & -0.08 & -0.13 \\
Collagen & -0.04 & -0.12 & -0.14 & 0.40 & -0.08 & -0.36 \\
Elastin & 0.09 & 0.12 & 0.01 & 0.11 & -0.01 & -0.24 \\
Nucleus & -0.26 & -0.17 & 0.02 & -0.03 & -0.04 & 0.20 \\
Keratin & -0.49 & 0.09 & 0.06 & -0.27 & -0.06 & 0.55 \\
Ceramide & 0.06 & 0.45 & 0.13 & -0.05 & -0.29 & 0.00 \\
Water & -0.54 & 0.03 & 0.09 & -0.14 & 0.09 & 0.37 \\
\hline
\end{tabular}

Other than the trends observed in tumor groups, we also recorded strong/moderate correlations in healthy skin structures such as fat vs. triolein, dermis vs. collagen, and sebaceous glands vs. ceramide (Figure $3 \mathrm{~d}-\mathrm{f}$ ). These correlations reflect our understanding of the skin's structural components as triolein is an important constituent in fat, collagen makes up the dermis, and sebaceous glands contain large amounts ceramide [24]. Our findings align and complement preceding studies $[37,38]$ that examined the distinctive Raman band differences between malignant skin lesions and normal skin. Gniadecka et al. observed an increased intensity of the band around $1000 \mathrm{~cm}^{-1}$ from the phenyl ring in SCC due to hyperkeratosis as phenyl ring structure is abundant in the keratin molecule [37]. Gniadecka et al. also reported a decreased intensity of the band $1271 \mathrm{~cm}^{-1}$ of amide III of proteins (collagen, elastin) in SCC, which is consistent with our finding of the reduced elastin and collagen due to extracellular matrix reorganization in malignant tissue. By translating the spectral difference of Raman spectra into biochemical contribution differences, our study complements earlier studies by directly assigning these bands to biophysical tissue constituents. 

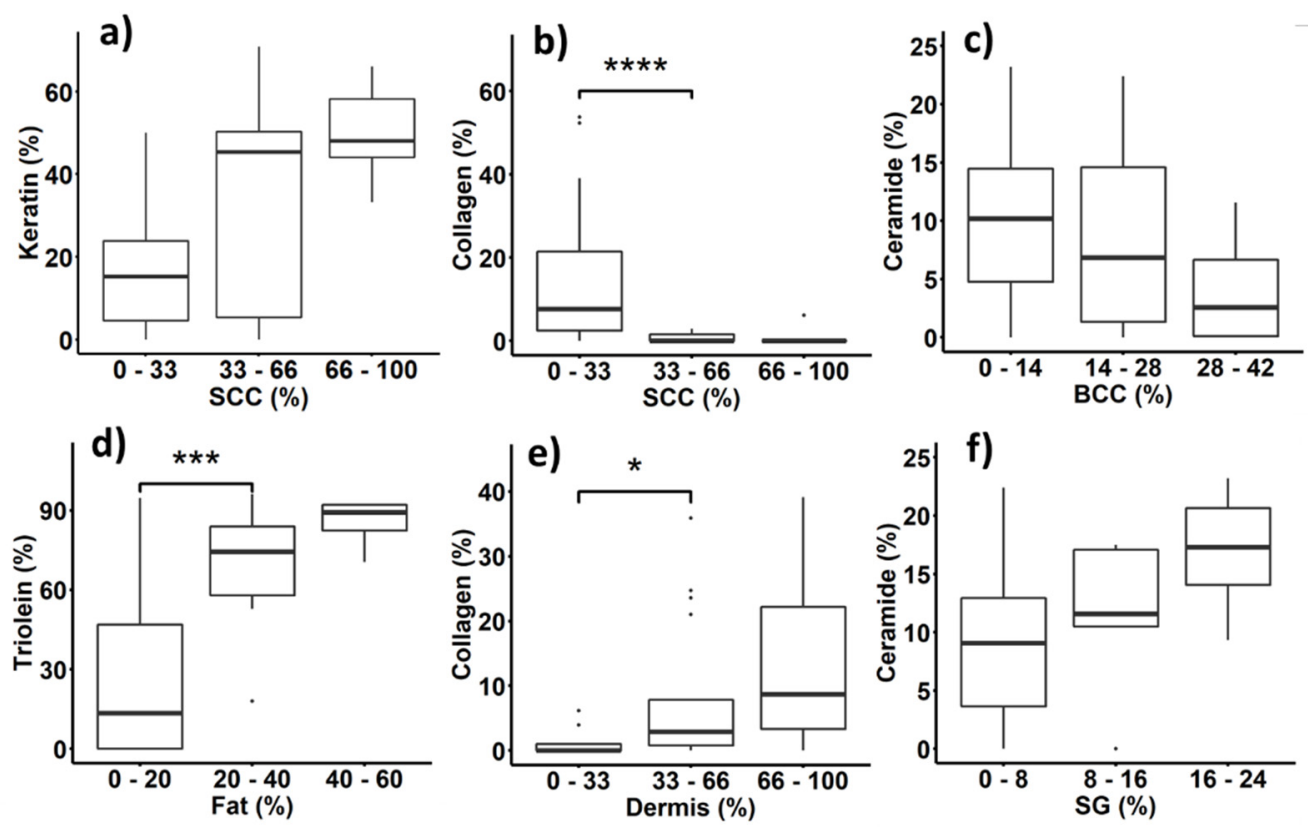

Figure 3. Contribution of biomarkers extracted from Raman spectra versus the percentage of tissue structures from histological analysis (total number of measurements is 82) in (a) Keratin vs. SCC, (b) Collagen vs. SCC, (c) Ceramide vs. BCC, (d) Triolein vs. Fat, (e) Collagen vs. Dermis and (f) Ceramide vs. SG. The percentages of tissue structures were divided equally into three sections for distribution comparison. Statistical analysis was performed via analysis of variance (ANOVA) combined with Tukey's test as a post hoc analysis. Statistical significance notion: ${ }^{*} p \leq 0.05,{ }^{* *} p \leq 0.01,{ }^{* * *} p \leq 0.001$, $* * * * p \leq 0.0001$.

We demonstrated statistical comparisons of each biomarker's distribution between tumor groups from the normal group in Figure 4. The SCC group had significantly higher keratin and nucleus contributions and less contribution in collagen than the normal group. The BCC group has a significantly lower level of ceramide than the normal group. These observations in BCC and SCC are consistent with our findings from Table 2. Additional analysis to distinguish tumor (BCC or SCC) and normal group with cross validation and receiver operating characteristic curve was presented in Figure S1 (Supplemental Materials).

One limitation of this study is that we used single H\&E-stained cross-section images to correlate to the Raman signals collected from the tissue volume. Future studies should consider several H\&E sections spaced apart to enable volumetric reconstruction of the sampling volume of the measurement, which will result in a more precise correlation between the Raman signal and underlying tissue. Another limitation of this study is that it simplified the model to only seven Raman active components. We originally reported a skin model with fifteen components in a prior study, but we decided to reduce the number of components to seven to avoid collinearity issues and reduce fitting variance [25]. Regardless, we acknowledged that there are far more molecules in the skin, and our seven-component-model may underestimate the contribution of other molecules to the Raman signal. The biophysical model we used in this study was built based on seven skin active biomarkers with different Raman scattering cross-sections. Several biomarkers that pathologically correlated with tumors (nucleus, collagen, and elastin) had lower Raman cross-sections than triolein. Hence, the detectability of nucleus, collagen, and elastin can be limited or sometimes overwhelmed by the triolein in the sampling volume. The biophysical model used in this study contains seven biomarkers, including collagen, elastin, triolein, nucleus, keratin, ceramide, and water, that are suitable for excised skin tissue. For future intraoperative studies, additional biomarkers such as plasma and blood cells should be added to the biophysical model to account for the operative condition. Another consideration for future research is to examine the high wavenumber region of the skin 
tissue Raman spectra (2400-3800 $\left.\mathrm{cm}^{-1}\right)$. Previous studies have shown high cancer detection capabilities when using the high wavenumber region alone [39] or in combination with the fingerprint region $\left(400-1800 \mathrm{~cm}^{-1}\right)$ [40].

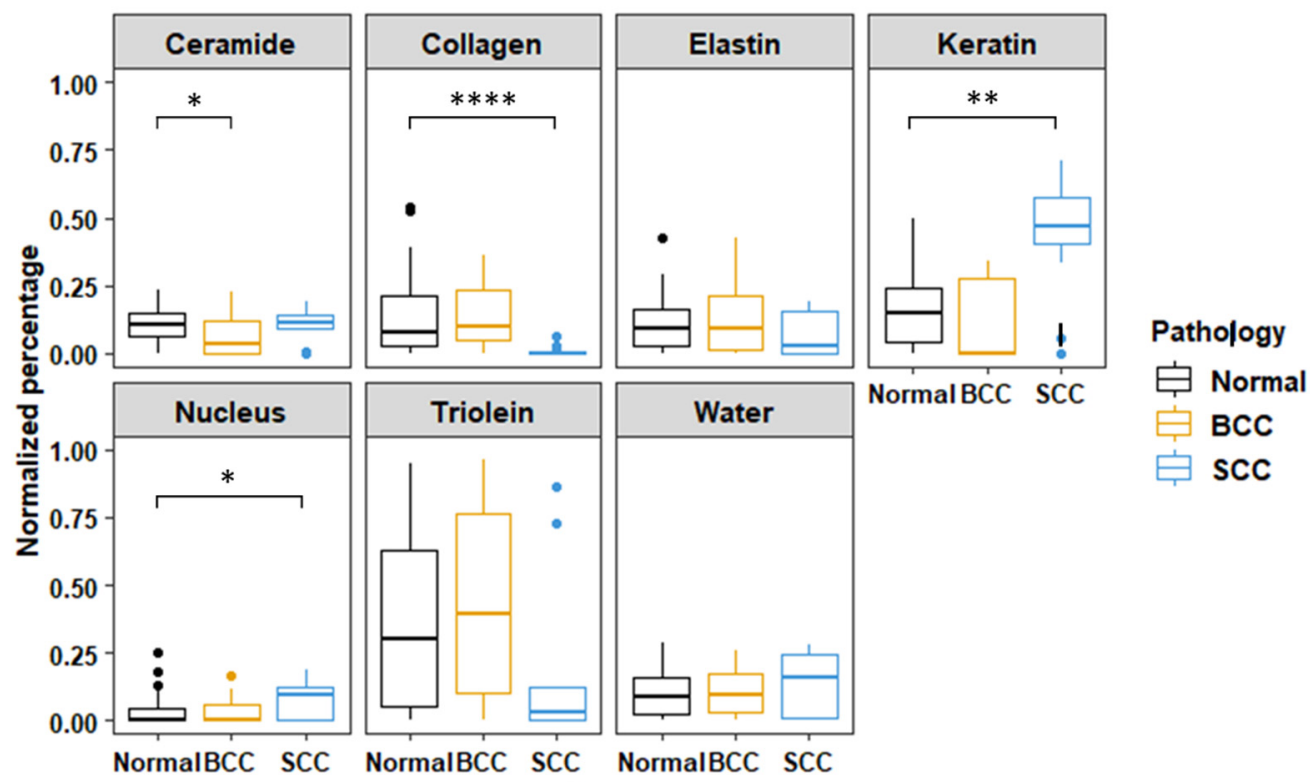

Figure 4. Statistical analysis to compare differences in the contribution of biomarkers in the tumor (BCC or SCC) and normal skin (including the dermis, hair follicles, sebaceous glands, and fat) Statistical analysis was performed via analysis of variance (ANOVA) combined with Tukey's test as a post hoc analysis. Statistical significance notion: ${ }^{*} p \leq 0.05,{ }^{* *} p \leq 0.01,{ }^{* * *} p \leq 0.001,{ }^{* * *} p \leq 0.0001$.

\section{Conclusions}

We used a fiber probe to acquire Raman spectra on ex vivo skin tissue from Mohs surgery patients. Through a biophysical model previously developed by our group, we extracted the contribution of critical biomarkers in the skin from the Raman spectra. We then examined the correlations between the biomarkers and the major skin structures (including the dermis, sebaceous glands, hair follicles, fat, BCC, and SCC) within the sampling area. We observed strong correlations in healthy skin structures such as fat vs. triolein, dermis vs. collagen, and sebaceous glands vs. ceramide. Our results also showed that SCC had a significantly different concentration of keratin, collagen, and nucleus when compared to normal structures, while BCC showed lower amounts of ceramide than normal structures. Our findings demonstrate the ability of Raman spectroscopy to measure skin biophysical contribution, which varies greatly across skin structures and pathologies. This information may impact future studies and approaches using Raman spectroscopy as diagnostic and surgical guidance tools.

Supplementary Materials: The following are available online at https:/ /www.mdpi.com/article/10 .3390 / photonics8070282/s1, Figure S1: Additional analysis to distinguish tumor (BCC or SCC) and normal group.

Author Contributions: Conceptualization, J.W.T. and H.T.M.N.; methodology, J.W.T. and H.T.M.N.; formal analysis, H.T.M.N. and Y.Z.; investigation, H.T.M.N., Y.Z., A.J.M., X.F., K.R.S. and M.C.F.; resources, J.W.T., M.K.M., J.S.R. and M.C.F.; writing—original draft preparation, H.T.M.N.; writingreview and editing, J.W.T., M.K.M. and H.T.M.N.; funding acquisition, J.W.T. and J.S.R. All authors have read and agreed to the published version of the manuscript.

Funding: This research was funded by the Cancer Prevention and Research Institute of Texas (CPRIT RP130702) and Texas Health Catalyst from The University of Texas at Austin. 
Institutional Review Board Statement: The study was conducted according to the guidelines of the Declaration of Helsinki, and approved by the Institutional Review Board (or Ethics Committee) of The University of Texas at Austin and the Seton Healthcare Family (\#2013-07-0008, approved on 08/26/2013).

Informed Consent Statement: Informed consent was obtained from all subjects involved in the study.

Data Availability Statement: The data presented in this study are available on request from the corresponding author.

Acknowledgments: The authors would like to thank the Austin Dermatological Surgical Center's staff, physician assistants, and physicians for their kind help with the skin tissue samples. We appreciate the time and effort of Sandra Esparza and Leandra Turner in preparing samples.

Conflicts of Interest: The authors declare no conflict of interest.

\section{References}

1. Nguyen, H.T.M.; Moy, A.J.; Zhang, Y.; Feng, X.; Reichenberg, J.S.; Fox, M.; Tunnell, J.W. Tumor Margin Assessment in Mohs Surgery Using Reflectance, Fluorescence and Raman Spectroscopy. In Proceedings of the Advanced Biomedical and Clinical Diagnostic and Surgical Guidance Systems X.V., San Francisco, CA, USA, 29-31 January 2017; Volume 10054, p. 1005403.

2. Zhang, Y.; Moy, A.J.; Feng, X.; Nguyen, H.T.M.; Sebastian, K.R.; Reichenberg, J.S.; Wilke, C.O.; Markey, M.K.; Tunnell, J.W. Assessment of Raman Spectroscopy for Reducing Unnecessary Biopsies for Melanoma Screening. Molecules 2020, $25,2852$. [CrossRef] [PubMed]

3. Feng, X.; Moy, A.J.; Nguyen, H.T.M.; Zhang, Y.; Zhang, J.; Fox, M.C.; Sebastian, K.R.; Reichenberg, J.S.; Markey, M.K.; Tunnell, J.W. Raman Biophysical Markers in Skin Cancer Diagnosis. JBO 2018, 23, 057002. [CrossRef]

4. Schmälzlin, E.; Moralejo, B.; Gersonde, I.; Schleusener, J.; Darvin, M.E.; Thiede, G.; Roth, M.M. Nonscanning Large-Area Raman Imaging for Ex Vivo/In Vivo Skin Cancer Discrimination. JBO 2018, 23, 105001. [CrossRef]

5. Silveira, L., Jr.; Silveira, F.L.; Pacheco, M.T.; Bodanese, B. Discriminating Model for Diagnosis of Basal Cell Carcinoma and Melanoma In Vitro Based on the Raman Spectra of Selected Biochemicals. JBO 2012, 17, 077003. [CrossRef]

6. Behl, I.; Kukreja, L.; Deshmukh, A.; Singh, S.P.; Mamgain, H.; Hole, A.R.; Krishna, C.M. Raman Mapping of Oral Buccal Mucosa: A Spectral Histopathology Approach. JBO 2014, 19, 126005. [CrossRef] [PubMed]

7. Cals, F.L.J.; Schut, T.C.B.; Caspers, P.J.; de Jong, R.J.B.; Koljenović, S.; Puppels, G.J. Raman Spectroscopic Analysis of the Molecular Composition of Oral Cavity Squamous Cell Carcinoma and Healthy Tongue Tissue. Analyst 2018, 143, 4090-4102. [CrossRef] [PubMed]

8. Haka, A.S.; Volynskaya, Z.; Gardecki, J.A.; Nazemi, J.; Lyons, J.; Hicks, D.; Fitzmaurice, M.; Dasari, R.R.; Crowe, J.P.; Feld, M.S. In Vivo Margin Assessment during Partial Mastectomy Breast Surgery Using Raman Spectroscopy. Cancer Res. 2006, 66, 3317-3322. [CrossRef] [PubMed]

9. Keller, M.D.; Vargis, E.; de Matos Granja, N.; Wilson, R.H.; Mycek, M.-A.; Kelley, M.C.; Mahadevan-Jansen, A. Development of a Spatially Offset Raman Spectroscopy Probe for Breast Tumor Surgical Margin Evaluation. J. Biomed. Opt. 2011, 16. [CrossRef]

10. Kirsch, M.; Schackert, G.; Salzer, R.; Krafft, C. Raman Spectroscopic Imaging for in Vivo Detection of Cerebral Brain Metastases. Anal. Bioanal. Chem. 2010, 398, 1707-1713. [CrossRef]

11. Jermyn, M.; Mok, K.; Mercier, J.; Desroches, J.; Pichette, J.; Saint-Arnaud, K.; Bernstein, L.; Guiot, M.-C.; Petrecca, K.; Leblond, F. Intraoperative Brain Cancer Detection with Raman Spectroscopy in Humans. Sci. Transl. Med. 2015, 7, 274ra19. [CrossRef]

12. Magee, N.D.; Villaumie, J.S.; Marple, E.T.; Ennis, M.; Elborn, J.S.; McGarvey, J.J. Ex Vivo Diagnosis of Lung Cancer Using a Raman Miniprobe. J. Phys. Chem. B 2009, 113, 8137-8141. [CrossRef]

13. Bourbousson, M.; Soomro, I.; Baldwin, D.; Notingher, I. Ex Vivo Raman Spectroscopy Mapping of Lung Tissue: Label-Free Molecular Characterization of Nontumorous and Cancerous Tissues. J. Med. Imaging 2019, 6, 036001. [CrossRef]

14. Teh, S.K.; Zheng, W.; Ho, K.Y.; Teh, M.; Yeoh, K.G.; Huang, Z. Diagnostic Potential of Near-Infrared Raman Spectroscopy in the Stomach: Differentiating Dysplasia from Normal Tissue. Br. J. Cancer 2008, 98, 457-465. [CrossRef] [PubMed]

15. Wan, Q.-S.; Wang, T.; Zhang, K.-H. Biomedical Optical Spectroscopy for the Early Diagnosis of Gastrointestinal Neoplasms. Tumour Biol. 2017, 39. [CrossRef] [PubMed]

16. Lieber, C.A.; Majumder, S.K.; Ellis, D.L.; Billheimer, D.D.; Mahadevan-Jansen, A. In-Vivo Nonmelanoma Skin Cancer Diagnosis Using Raman Microspectroscopy. Lasers Surg. Med. 2008, 40, 461-467. [CrossRef] [PubMed]

17. Lim, L.; Nichols, B.; Migden, M.R.; Rajaram, N.; Reichenberg, J.S.; Markey, M.K.; Ross, M.I.; Tunnell, J.W. Clinical Study of Noninvasive in vivo Melanoma and Nonmelanoma Skin Cancers Using Multimodal Spectral Diagnosis. J. Biomed. Opt. 2014, 19, 117003. [CrossRef] [PubMed]

18. Ferreira Lima, A.M.; Daniel, C.R.; Navarro, R.S.; Bodanese, B.; Pasqualucci, C.A.; Tavares Pacheco, M.T.; Zângaro, R.A.; Silveira, L. Discrimination of Non-Melanoma Skin Cancer and Keratosis from Normal Skin Tissue in Vivo and Ex Vivo by Raman Spectroscopy. Vib. Spectrosc. 2019, 100, 131-141. [CrossRef] 
19. Silveira, F.L.; Pacheco, M.T.T.; Bodanese, B.; Pasqualucci, C.A.; Zângaro, R.A.; Silveira, L. Discrimination of Non-Melanoma Skin Lesions from Non-Tumor Human Skin Tissues in Vivo Using Raman Spectroscopy and Multivariate Statistics. Lasers Surg. Med. 2015, 47, 6-16. [CrossRef]

20. Nijssen, A.; Maquelin, K.; Santos, L.F.; Caspers, P.J.; Schut, T.C.B.; den Hollander, J.C.; Neumann, M.H.A.; Puppels, G.J. Discriminating Basal Cell Carcinoma from Perilesional Skin Using High Wave-Number Raman Spectroscopy. JBO 2007, 12, 034004. [CrossRef] [PubMed]

21. Schleusener, J.; Gluszczynska, P.; Reble, C.; Gersonde, I.; Helfmann, J.; Fluhr, J.W.; Lademann, J.; Röwert-Huber, J.; Patzelt, A.; Meinke, M.C. In Vivo Study for the Discrimination of Cancerous and Normal Skin Using Fibre Probe-Based Raman Spectroscopy. Exp. Dermatol. 2015, 24,767-772. [CrossRef]

22. Bratchenko, I.A.; Bratchenko, L.A.; Moryatov, A.A.; Khristoforova, Y.A.; Artemyev, D.N.; Myakinin, O.O.; Orlov, A.E.; Kozlov, S.V.; Zakharov, V.P. In Vivo Diagnosis of Skin Cancer with a Portable Raman Spectroscopic Device. Exp. Dermatol. 2021, 30, 652-663. [CrossRef]

23. Zhao, J.; Lui, H.; McLean, D.I.; Zeng, H. Real-Time Raman Spectroscopy for Non-Invasive Skin Cancer Detection-Preliminary Results. In Proceedings of the 2008 30th Annual International Conference of the IEEE Engineering in Medicine and Biology Society, Vancouver, BC, Canada, 20-25 August 2008; pp. 3107-3109. [CrossRef]

24. Feng, X.; Fox, M.C.; Reichenberg, J.S.; Lopes, F.C.P.S.; Sebastian, K.R.; Markey, M.K.; Tunnell, J.W. Biophysical Basis of Skin Cancer Margin Assessment Using Raman Spectroscopy. Biomed. Opt. Express 2019, 10, 104. [CrossRef]

25. Feng, X.; Moy, A.J.; Nguyen, H.T.M.; Zhang, J.; Fox, M.C.; Sebastian, K.R.; Reichenberg, J.S.; Markey, M.K.; Tunnell, J.W. Raman Active Components of Skin Cancer. Biomed. Opt. Express BOE 2017, 8, 2835-2850. [CrossRef]

26. Moy, A.J.; Feng, X.; Markey, M.K.; Reichenberg, J.S.; Tunnell, J.W. Noninvasive Skin Cancer Diagnosis Using Multimodal Optical Spectroscopy. In Proceedings of the Photonic Therapeutics and Diagnostics XII, San Francisco, CA, USA, 13 February 2016; Volume 9689, p. 968905.

27. Moy, A.J.; Feng, X.; Nguyen, H.T.M.; Zhang, Y.; Sebastian, K.R.; Reichenberg, J.S.; Tunnell, J.W. Spectral Biopsy for Skin Cancer Diagnosis: Initial Clinical Results. In Proceedings of the Photonics in Dermatology and Plastic Surgery, San Francisco, CA, USA, 9 February 2017; Volume 10037, p. 1003704.

28. Sharma, M.; Marple, E.; Reichenberg, J.; Tunnell, J.W. Design and Characterization of a Novel Multimodal Fiber-Optic Probe and Spectroscopy System for Skin Cancer Applications. Rev. Sci. Instrum. 2014, 85, 083101. [CrossRef] [PubMed]

29. Hennessy, R.; Goth, W.; Sharma, M.; Markey, M.K.; Tunnell, J.W. Effect of Probe Geometry and Optical Properties on the Sampling Depth for Diffuse Reflectance Spectroscopy. JBO 2014, 19, 107002. [CrossRef]

30. Koslosky, C.L.; El Tal, A.K.; Workman, B.; Tamim, H.; Durance, M.C.; Mehregan, D.A. Reliability of Skin Biopsies in Determining Accurate Tumor Margins: A Retrospective Study After Mohs Micrographic Surgery. Dermatol. Surg. 2014, 40, 964-970. [CrossRef]

31. Larraona-Puy, M.; Ghita, A.; Zoladek, A.; Perkins, W.; Varma, S.; Leach, I.H.; Koloydenko, A.A.; Williams, H.; Notingher, I. Discrimination between Basal Cell Carcinoma and Hair Follicles in Skin Tissue Sections by Raman Micro-Spectroscopy. J. Mol. Struct. 2011, 993, 57-61. [CrossRef]

32. Larraona-Puy, M.; Ghita, A.; Zoladek, A.B.; Perkins, W.; Varma, S.; Leach, I.H.; Koloydenko, A.; Williams, H.; Notingher, I. Development of Raman Microspectroscopy for Automated Detection and Imaging of Basal Cell Carcinoma. JBO 2009, 14, 054031. [CrossRef] [PubMed]

33. Yanofsky, V.R.; Mercer, S.E.; Phelps, R.G. Histopathological Variants of Cutaneous Squamous Cell Carcinoma: A Review. J. Ski. Cancer 2011, 2011, 210813. [CrossRef] [PubMed]

34. Henke, E.; Nandigama, R.; Ergün, S. Extracellular Matrix in the Tumor Microenvironment and Its Impact on Cancer Therapy. Front. Mol. Biosci. 2020, 6. [CrossRef] [PubMed]

35. Johnston, R.B. Tumors of the Epidermis. In Weedon's Skin Pathology Essentials; Elsevier: Amsterdam, The Netherlands, 2017; pp. 504-524. ISBN 978-0-7020-6830-0.

36. Choi, M.J.; Maibach, H.I. Role of Ceramides in Barrier Function of Healthy and Diseased Skin. Am. J. Clin. Dermatol. 2005, 6, 215-223. [CrossRef]

37. Gniadecka, M.; Wulf, H.C.; Nielsen, O.F.; Christensen, D.H.; Hercogova, J. Distinctive Molecular Abnormalities in Benign and Malignant Skin Lesions: Studies by Raman Spectroscopy. Photochem. Photobiol. 1997, 66, 418-423. [CrossRef] [PubMed]

38. Gniadecka, M.; Wulf, H.C.; Mortensen, N.N.; Nielsen, O.F.; Christensen, D.H. Diagnosis of Basal Cell Carcinoma by Raman Spectroscopy. J. Raman Spectrosc. 1997, 28, 125-129. [CrossRef]

39. Koljenovic, S.; Schut, T.C.B.; Wolthuis, R.; de Jong, B.; Santos, L.; Caspers, P.J.; Kros, J.M.; Puppels, G.J. Tissue Characterization Using High Wave Number Raman Spectroscopy. JBO 2005, 10, 031116. [CrossRef] [PubMed]

40. Aubertin, K.; Desroches, J.; Jermyn, M.; Trinh, V.Q.; Saad, F.; Trudel, D.; Leblond, F. Combining High Wavenumber and Fingerprint Raman Spectroscopy for the Detection of Prostate Cancer during Radical Prostatectomy. Biomed. Opt. Express 2018, 9, $4294-4305$. [CrossRef] 